Supporting Information

\title{
Activation on Ammonia Absorbing Reaction for Magnesium Chloride
}

Taihei Aoki ${ }^{1}$, Hiroki Miyaoka ${ }^{2}$, Hitoshi Inokawa ${ }^{2}$, Takayuki Ichikawa*3,4 and Yoshitsugu Kojima $^{4}$

${ }^{1}$ Graduate School of Advanced Sciences of Matter, Hiroshima University, 1-3-1

Kagamiyama, Higashi-Hiroshima, 739-8530, Japan

${ }^{2}$ Institute for Sustainable Sciences and Development, Hiroshima University, 1-3-1

Kagamiyama, Higashi-Hiroshima, 739-8530, Japan

${ }^{3}$ Graduate School of Integrated Arts and Sciences, Hiroshima University, 1-7-1

Kagamiyama, Higashi-Hiroshima, 739-8521, Japan

${ }^{4}$ Institute for Advanced Materials Research, Hiroshima University, 1-3-1 Kagamiyama, Higashi-Hiroshima, 739-8530, Japan

Table S1. Thermodynamics properties of ammine complex and relation material of that.

\begin{tabular}{lcc}
\hline & $\Delta_{f} H^{0}[\mathrm{~kJ} / \mathrm{mol}]$ & $S^{0}[\mathrm{~J} / \mathrm{mol} \mathrm{K}]$ \\
\hline $\mathrm{Mg}\left(\mathrm{NH}_{3}\right) \mathrm{Cl}_{2}$ & -752 & 185 \\
$\mathrm{Mg}\left(\mathrm{NH}_{3}\right)_{6} \mathrm{Cl}_{2}$ & -1265 & 352 \\
$\mathrm{MgCl}_{2}$ & $-642^{\mathrm{a}}$ & $90^{\mathrm{a}}$ \\
$\mathrm{NH}_{3}$ & $-46^{\mathrm{a}}$ & $193^{\mathrm{a}}$ \\
\hline
\end{tabular}

${ }^{a}$ NIST-JANAF Themochemical Tables. ${ }^{19}$ 


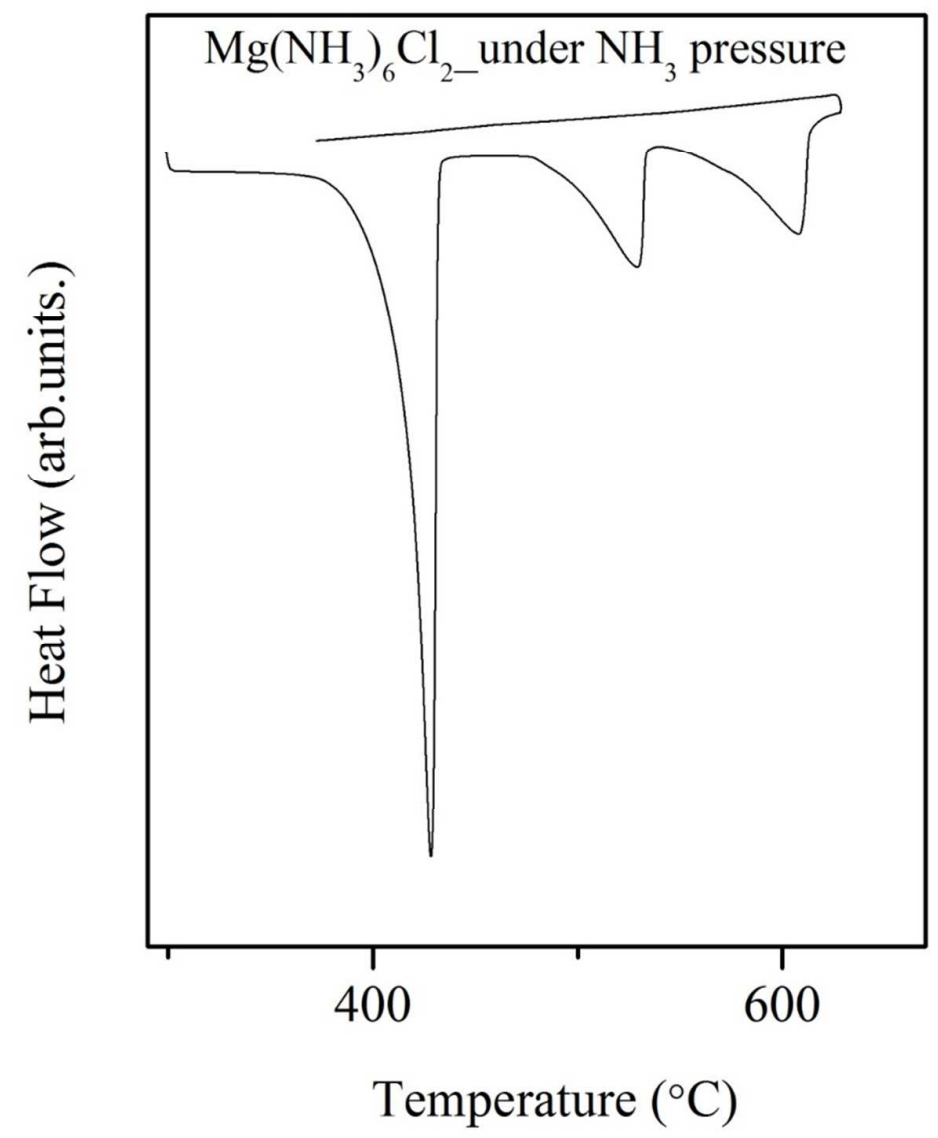

Figure S1. $\mathrm{NH}_{3}$ desorption profiles of $\mathrm{Mg}\left(\mathrm{NH}_{3}\right)_{6} \mathrm{Cl}_{2}$ under $\mathrm{NH}_{3}$ pressure. $\mathrm{NH}_{3}$ atmosphere was realized by the $\mathrm{NH}_{3}$ gas desorbed from the sample itself in closed sample pan. 


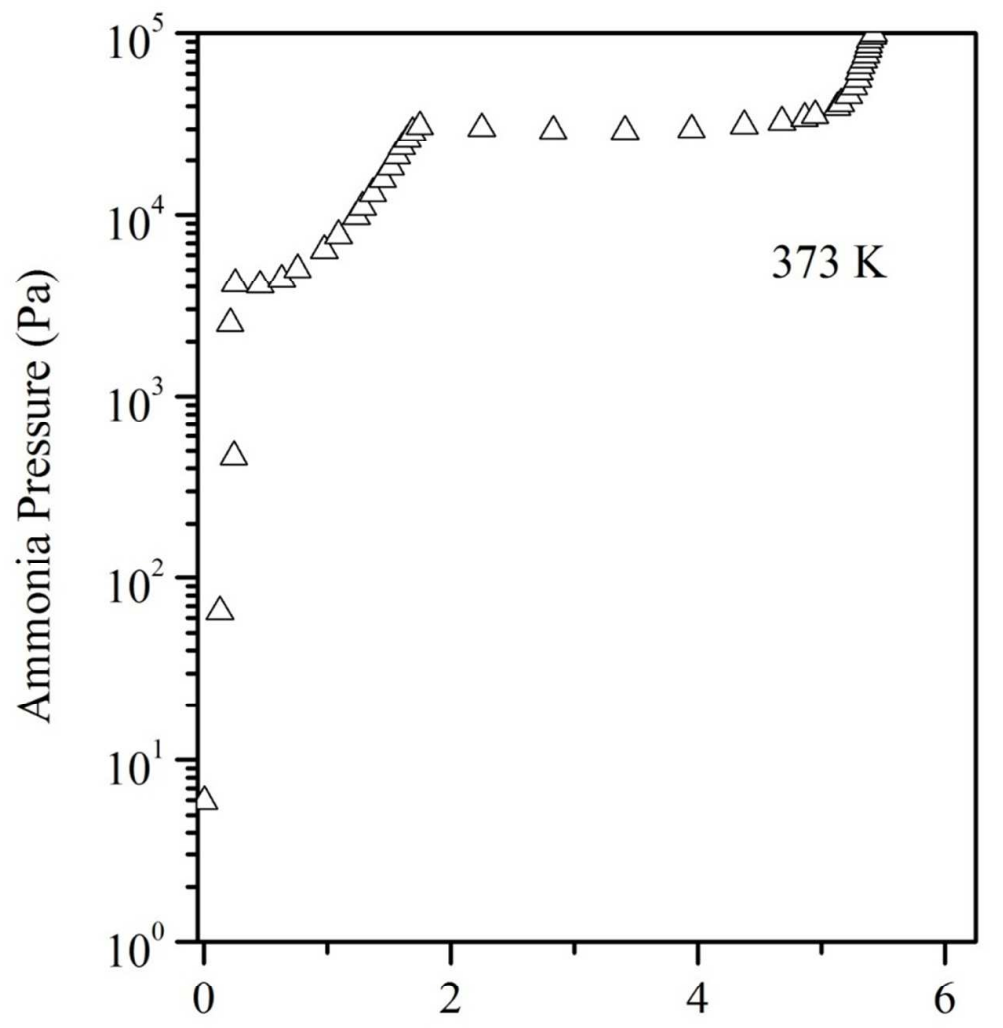

Amount of absorbed ammonia ( $\left.\mathrm{mol} / \mathrm{mol} \mathrm{MgCl}_{2}\right)$

Figure S2. PC isotherm for $\mathrm{NH}_{3}$ absorption of $\mathrm{MgCl}_{2}$ after $\mathrm{NH}_{3}$ treatment $373 \mathrm{~K}$. 


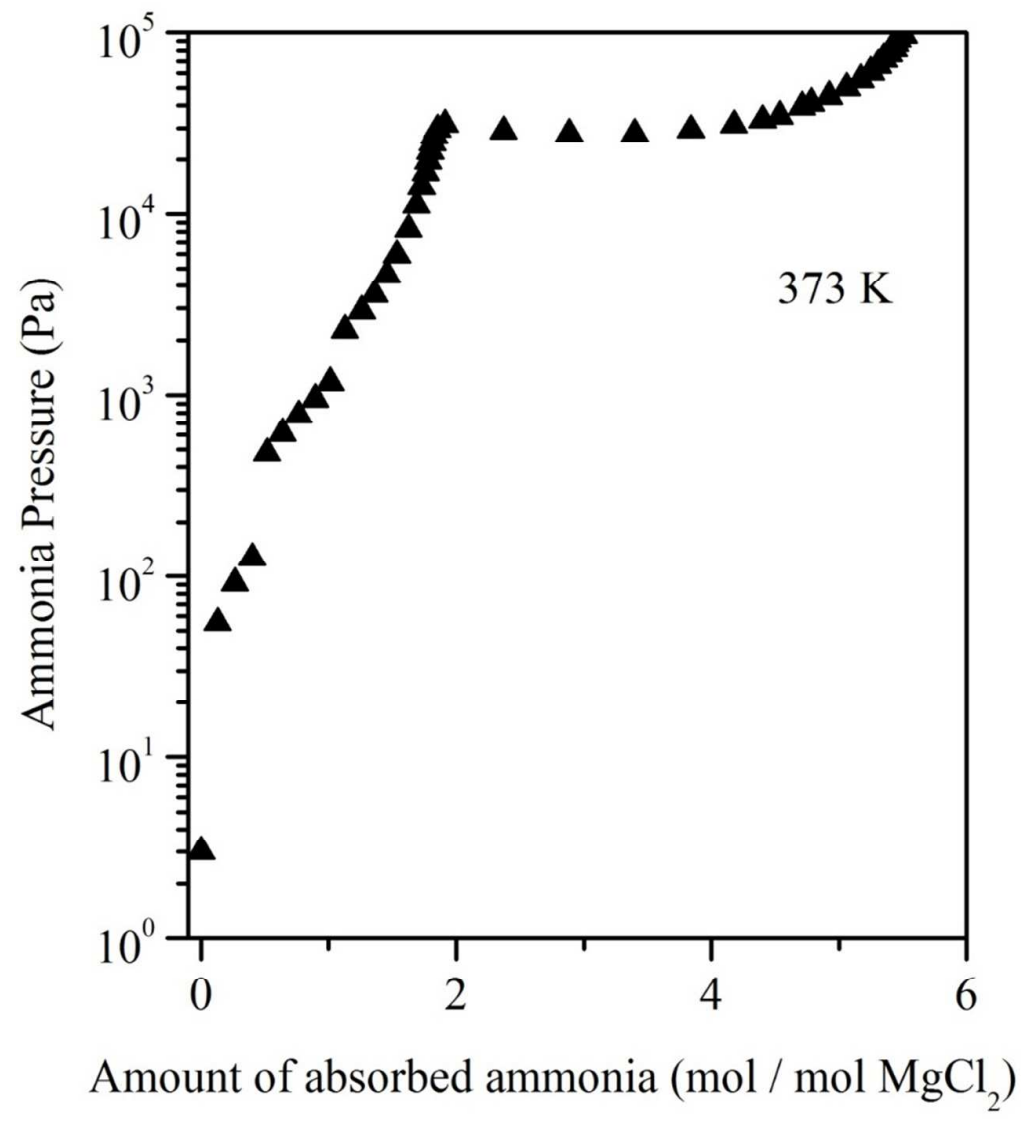

Figure S3. PC isotherm for $\mathrm{NH}_{3}$ absorption of $\mathrm{MgCl}_{2}$ after mechanical treatment at 373 K. 